\title{
La fête japonaise : appareil hégémonique de la cité marchande
}

The japanese festival: hegemonic device of the haggle town

\section{Shoichiro Takezawa}

\section{(2) OpenEdition}

12 Journals

Édition électronique

URL : https://journals.openedition.org/tc/5819

DOI : 10.4000/tc.5819

ISBN : 1952-420X

ISSN : 1952-420X

Éditeur

Éditions de l'EHESS

Édition imprimée

Date de publication : 15 décembre 2011

Pagination : 98-119

ISBN : 978-2-7351-1437-5

ISSN : 0248-6016

\section{Référence électronique}

Shoichiro Takezawa, "La fête japonaise : appareil hégémonique de la cité marchande », Techniques \& Culture [En ligne], 57 | 2011, mis en ligne le 30 juin 2012, consulté le 29 septembre 2022. URL : http:// journals.openedition.org/tc/5819; DOI : https://doi.org/10.4000/tc.5819 


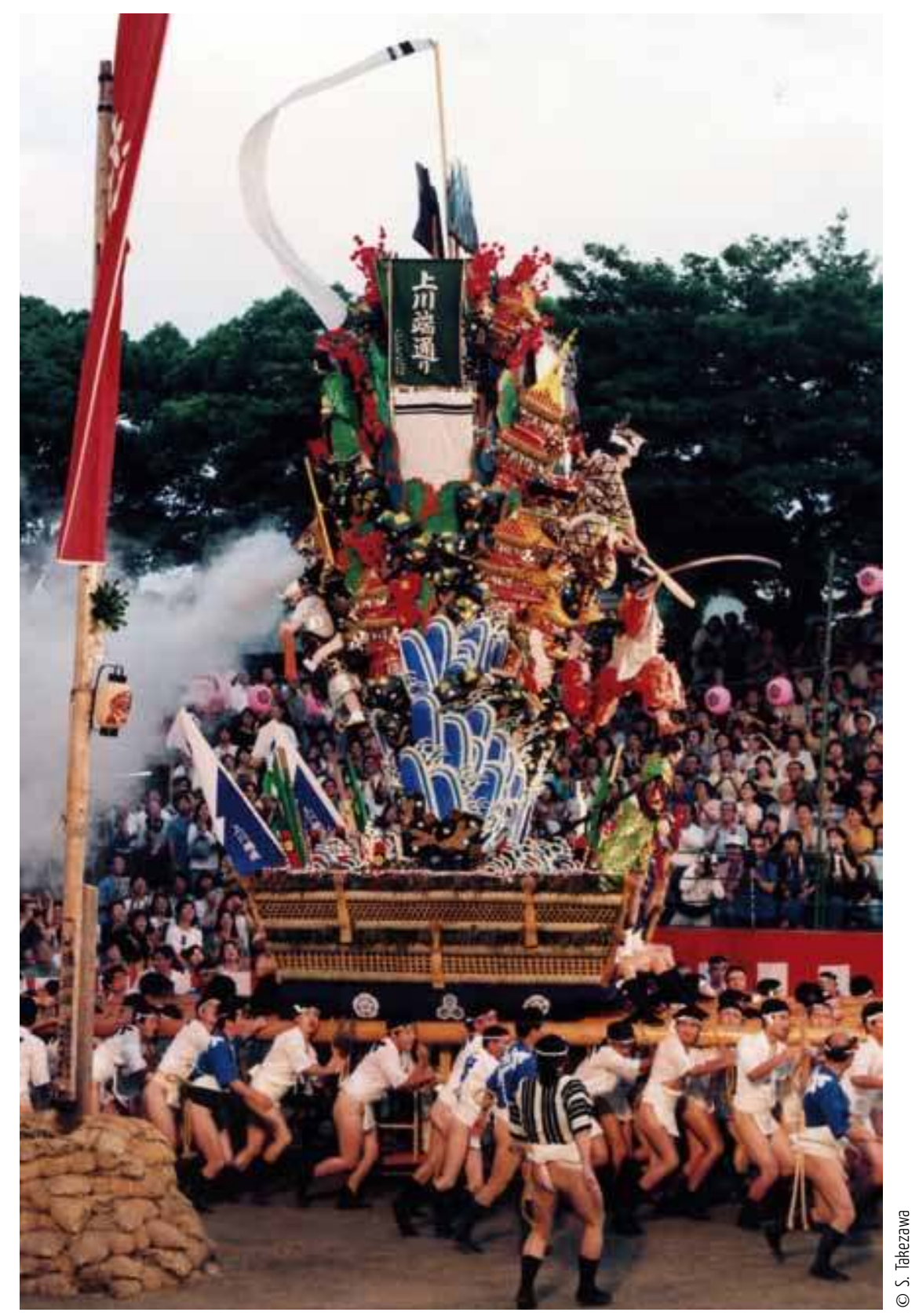




\section{LA FÊTE JAPONAISE}

\section{Appareil hégémonique de la cité marchande}

Les étrangers qui visitent le Japon lors de la fête du nouvel an s'étonneront de voir un grand nombre de Japonais aller prier dans les temples shintoïstes pour y faire des vœux de bonheur. Ce phénomène signifie-t-il pour autant que les Japonais soient des shintoïstes assidus? Certainement pas. En fait, si la plupart des Japonais se considèrent comme bouddhistes, ils ne négligent jamais, chaque année, de s'engager dans divers rites shinto, qu'il s'agisse de rites de passages (intervenant au cours de la vie) ou de rites accompagnant des activités culturelles tels que les rites agricoles, ou la fête annuelle.

Comment pouvons nous expliquer l'écart entre cette conscience d'appartenance à une croyance religieuse et une pratique distincte?

Nous pensons y voir la caractéristique du shintoïsme et d'autres systèmes religieux dits « ethniques » : faute de doctrine révélée par un dieu et l'existence d'un livre sacré, ces systèmes se forment principalement autour de pratiques ou de rituels effectués périodiquement par leurs adeptes. Les Japonais, en général, ne les considèrent pas comme des pratiques religieuses mais comme des pratiques coutumières non attachées, pensent-ils, à des croyances religieuses élaborées.

Plusieurs interrogations se posent par conséquent : pour quelle(s) raison(s) les Japonais s'engageaient-ils, et s'engagent-ils encore, dans les fêtes shintoïstes, alors qu'ils ne tiennent pas compte des croyances qui leur sont associées? Quelle est donc la mission que le shintoïsme peut remplir auprès de ceux qui ne manquent pas de le pratiquer?

Dans cet article nous aborderons ces questions en analysant la fête japonaise annuelle d'Hakata, ancienne cité marchande qui fait actuellement partie de la cité de Fukuoka. 


\section{Tableau de Hakata (en bas) et de Fukuoka (en haut)}

Sur ce tableau de la fin du xixe siècle, on peut voir une multitude de bateaux, petits et grands, ce qui indique que le port de Hakata était réputé tant pour le commerce extérieur que la pêche locale.
Cette fête dite gion yamakasa a lieu le 15 juillet (15 juin du calendrier lunaire, correspondant au solstice d'été) dans le but de débarrasser des mauvais sorts, tels que les épidémies ou les intoxications alimentaires, qui menaceraient la vie des citadins. Telle est la signification religieuse qui y est attribuée depuis longtemps. Mais beaucoup de participants, y compris les habitants locaux, en font peu de cas aujourd'hui. Ils s'y engagent, en considérant cette fête seulement comme un divertissement collectif d'été, ou une coutume traditionnelle.

Cet article s'organise en deux parties. La première sera consacrée à la description de l'histoire de la cité de Hakata et de sa fête : nous y démontrerons que l'armature de l'organisation sociale d'une cité marchande japonaise au temps pré-moderne était étroitement liée à son organisation religieuse ; nous y analyserons également l'impact que la contribution de la fête a pu avoir sur le développement social et économique de la cité. La seconde partie envisagera l'évolution de la fête au temps moderne. Depuis la Restauration de Meiji, les gouvernements centraux et locaux œuvrent à la constitution d'un État centralisé et sont intervenus dans l'organisation de la fête, effectuée jusqu'à cette période de façon autonome par la population locale.

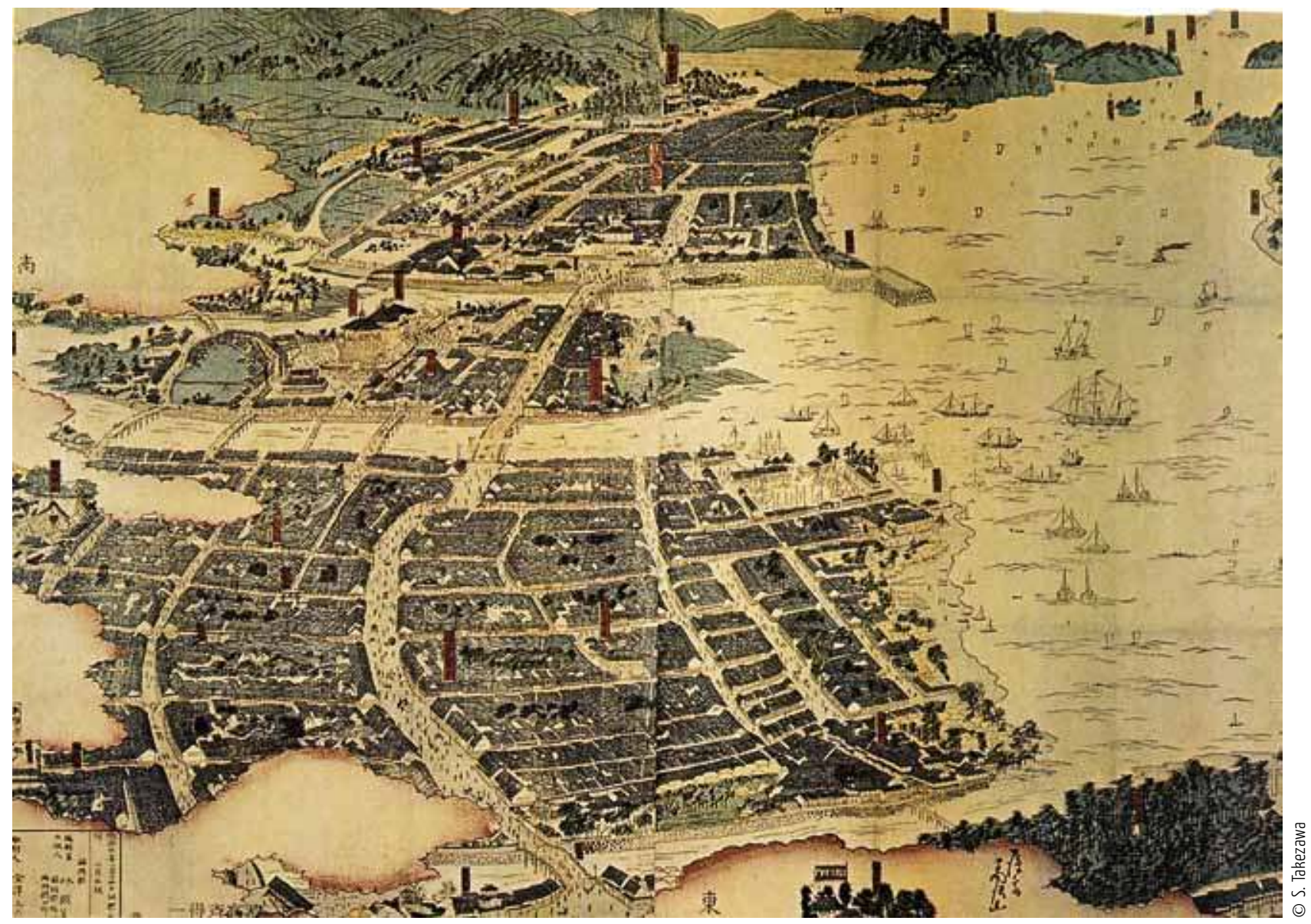


En analysant les conflits entre le gouvernement central et la société locale sur la question de l'organisation de la fête, nous voulons donner un nouvel éclairage du processus de transformation de la société japonaise.

\section{L'origine de Hakata et de sa fête}

Hakata, une des plus anciennes cités japonaises, se trouve au Sud-Ouest de l'archipel, en face de la péninsule coréenne. À l'époque de Nara (710-784), une maison spéciale appelée « Korokan» fut construite aux environs de Hakata, afin de recevoir les missions diplomatiques venant de Chine et de Corée. À cette époque cependant, les principales activités politiques et économiques couvrant le Sud-Ouest du Japon se déroulaient à Dazaifu, vingt kilomètres au sud de Hakata. Hakata n'était alors qu'une petite agglomération de pêcheurs. Sa construction en tant que cité marchande débuta à la fin de l'époque de Heian, sous le règne de Taira Kiyomori (1118-1181). Envisageant l'intensification du commerce extérieur avec la Chine, il encouragea le développement de Hakata. Un temple bouddhiste appelé «Shôhuku-ji » y fut construit en 1195, suivi d'un autre temple, « Jôten-ji » en 1242. Eisai, qui construisit le temple Shohuku-ji fut aussi le premier à introduire le zen et le thé au Japon. Ce prêtre bouddhiste était à la fois un rénovateur culturel et un commerçant international. Hakata se trouvait au carrefour des idées et des marchandises nouvelles venant de Chine, de Corée et de Kyoto.

Le développement de Hakata se poursuivit sans interruption. En 1480, un poète japonais appelé Sôgi écrivit à ce propos :

On peut remarquer au large [de Hakata] beaucoup de grands navires. [...] On n'en finit pas d'y compter le nombre des temples ni des prêtres bouddhistes. Les maisons des plébéiens se serrent les unes contre les autres sur une immense étendue (Kawazoe 1988 : 36).

Il ne faut pas voir dans cette description une expression isolée, improvisée par un voyageur. Un autre document de la même époque affirme que l'on comptait environ dix mille maisons (ibid. : 35) ; si l'on estime à huit le nombre de personnes dans chaque maison, cela nous donne une population totale de 80000 mille habitants. Ce chiffre montre que Hakata était une ville exceptionnellement grande et prospère en son temps.

Comme Hakata était une cité portuaire ouverte aux voyageurs, un grand nombre de commerçants étrangers y vinrent, dont des missionnaires Portugais. Ces derniers, savants de premier ordre, ne manquèrent pas de noter la prospérité de la cité, les coutumes et l'organisation sociale. Luis Frois (1537-1597), par exemple, qui resta au Japon pendant une trentaine d'années, admira la prospérité de Hakata dans son grand ouvrage sur l'histoire du Japon (Frois : 1976 (1) : 114-115) :

À cette époque, dans toutes les régions du Ximo (l'île de Kyushu), il n’y avait pas de cité aussi noble et aussi riche que Facata, dans toute la république des marchands, qui ne voulut imiter la noble cité de Sakai ${ }^{1}$. 
Si les activités missionnaires portugaises eurent un grand succès dans les autres cités de la même île, elles n'acquirent qu'une poignée d'adeptes à Hakata, alors même que cette dernière était ouverte aux étrangers et qu'il n'y avait aucun contrôle sur leurs activités. Les causes de cet insuccès doivent être recherchées dans l'assiduité que les citadins de Hakata attachaient à leurs pratiques religieuses traditionnelles. Frois jeta d'ailleurs un regard hostile envers ces pratiques.

Nous ne trouvons pas, par ailleurs, de grandes différences entre la fête du Xvi siècle et celle qui a lieu de nos jours. Ainsi, certaines caractéristiques de la fête décrites par Frois peuvent encore être observées aujourd'hui : l'appellation de la fête de gion, la construction annuelle des chars et leur défilé dans la ville, le rassemblement exalté des citadins, l'exercice de l'autorité des aînés sur les jeunes, etc. Certaines questions s'imposent néanmoins : comment l'organisation de la fête s'est-elle constituée? Qui l'a financée? Quel rôle cette fête a-t-elle joué pour le développement économique et social de la ville? Pour répondre à ces questions, nous devons prendre en compte l'organisation sociale de la cité de Hakata au temps pré-moderne. Cette dernière était alors étroitement liée à l'organisation religieuse à la base de cette très coûteuse fête annuelle.

\section{L'organisation sociale de la cité marchande de Hakata au temps pré-moderne}

Hier comme aujourd'hui, Hakata se déploie sur un terrain d'un kilomètre de long et d'un kilomètre de large entouré d'eau. Deux rivières la ceignent à l'est et à l'ouest. Au nord se trouve la baie de Hakata, autrefois propice aux trafics fluviaux. Au sud, un fossé artificiel fut creusé pour la protéger.

La prospérité de Hakata dépendait d'abord de sa situation géographique - en face de la péninsule coréenne. Cette position privilégiée fit de Hakata la cité marchande où aucun seigneur (samurai) n'essaya de régner de façon directe, sachant que les activités libres des commerçants lui procuraient de grands profits. En revanche, la cité devint la cible des seigneurs qui voulaient imposer leur tutelle pour en tirer des bénéfices. Hakata fut régulièrement attaquée durant la période de guerres intestines (1476-1600). Et ce fut un grand seigneur, Toyotomi Hideyohi, unificateur du Japon à la fin de cette période, qui rétablit l'ordre qui allait subsister durant toute l'époque d'Edo (1600-1868).

Ce seigneur rédigea un avis officiel composé de neuf chapitres, qui ordonnait les activités commerciales de la cité : interdiction de résidence pour le samurai, libre circulation des commerçants et libre échange des marchandises, baisse des impôts et exemption de la corvée. Il établit en même temps un plan de réaménagement urbain : il fit construire sept boulevards qui traversèrent la cité de long en large et fit édifier les sept temples Kan'on et sept temples Odô pour la prière journalière. Par là, Hideyoshi voulait structurer la vie matérielle et spirituelle des citadins. En effet, ces sept boulevards devinrent à la fois la base de l'infrastructure de Hakata et l'armature de la vie sociale et religieuse de ses habitants.

Àl'époque d'Edo, la base de la vie des citadins de Hakata résidait dans le cho (la communauté). Le cho se composait en moyenne d'une trentaine de maisons, c'est-à-dire d'environs deux cents cinquante personnes ${ }^{2}$. C'était dans le cho qu'une personne naissait, vivait et mourrait. 


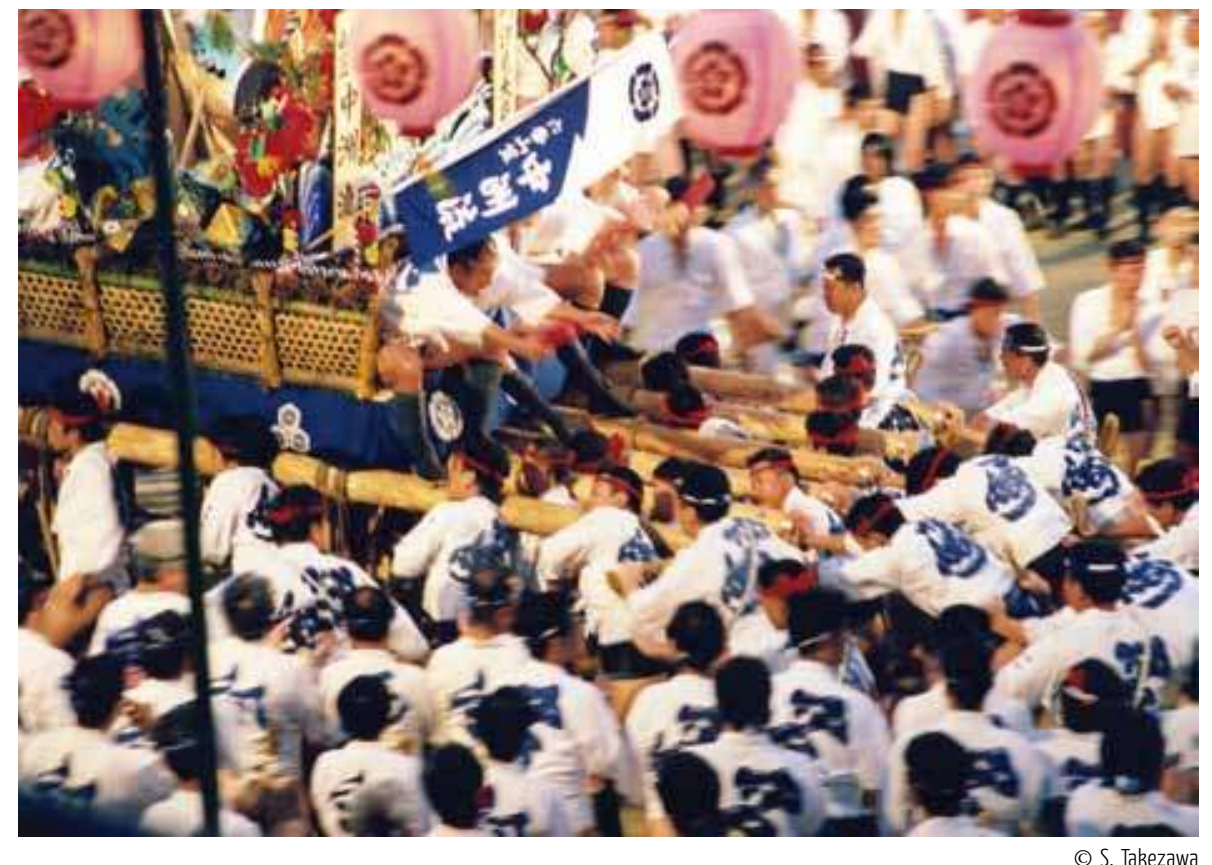

\section{Char de la fête de gion}

Afin de parcourir toute la ville de Hakata, il est porté par une trentaine d'hommes et poussé par une vingtaine. Comme un char pèse environ une tonne et que les hommes ne peuvent le porter que pendant 30

secondes, ceux qui le portent et le poussent se remplacent en courant. II faut en moyenne un millier de participants par char.

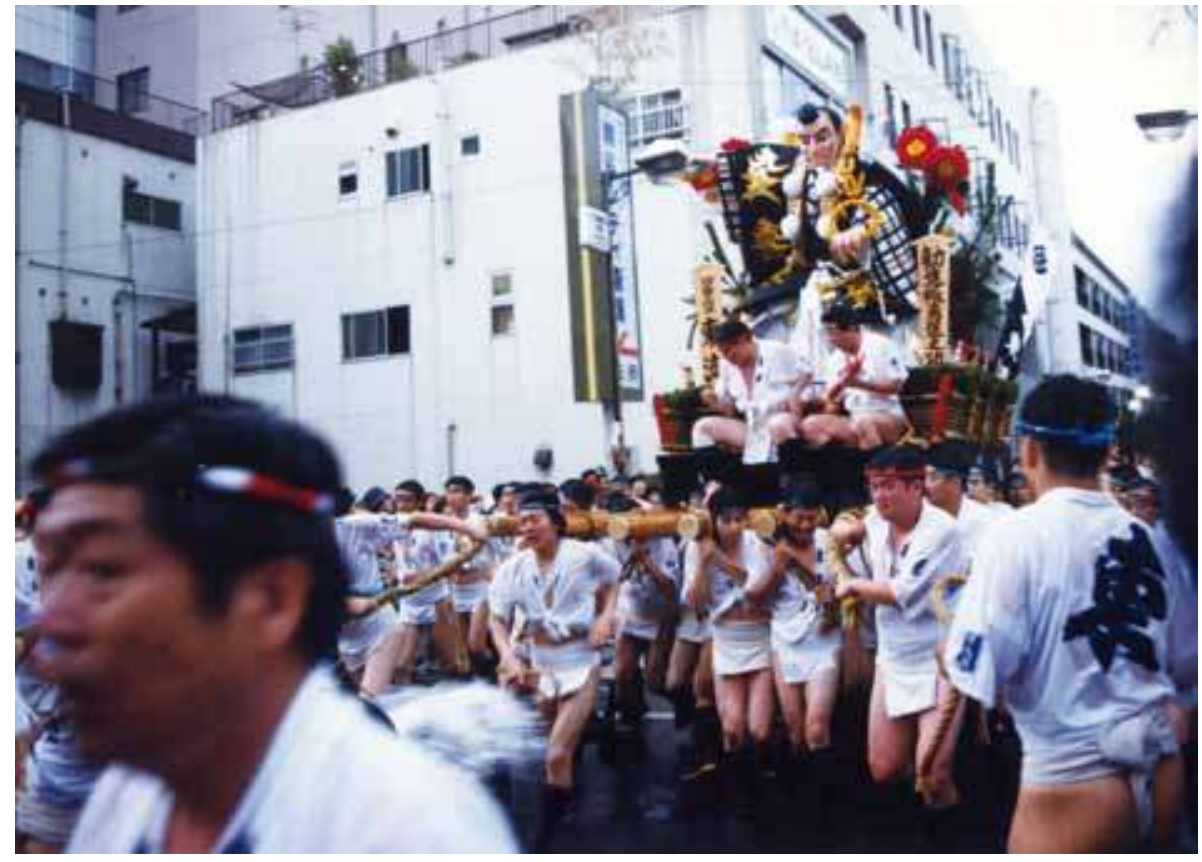

(c) S. Takezawa 
Le cho avait plusieurs fonctions : enregistrement de la naissance et de la mort de ses membres; publication de toutes sortes d'attestations ; fixation et collecte des impôts de chaque maison ; stockage du riz pour parer à l'imprévu ${ }^{3}$. Au temps pré-moderne, la classe dominante se contentait de collecter les impôts du peuple, en leur laissant une certaine autonomie ; mais en échange de cette autonomie, la population s'engageait à construire et maintenir l'infrastructure de la cité. C'était donc le cho qui prenait en charge le maintien et la réparation de la portion de boulevard, de rue, le pont et le port qui faisaient partie de son territoire. Le cho devait également gérer le puits commun et nettoyer le fossé pour évacuer les eaux d'égout. En cas d'incendie, c'était le groupe des jeunes de chaque cho qui devait l'éteindre. Enfin, à la frontière de chaque cho,

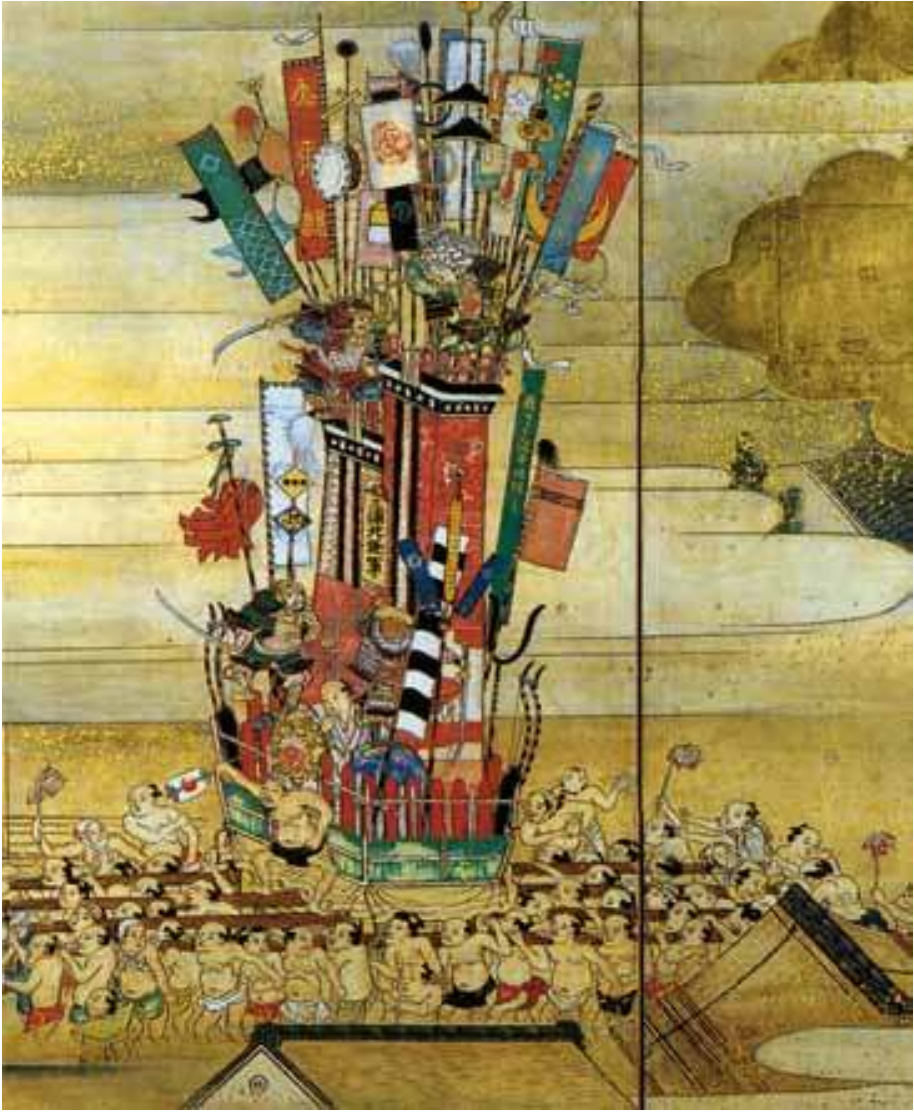

(C) S. Takezawa une petite porte était construite qui devait être fermée pendant la nuit ${ }^{4}$. On peut dire du cho qu'il était comme une grande maison dans laquelle tous les membres vivaient ensemble.

Si le cho constituait ainsi une unité économique et sociale pour les citadins de Hakata, il correspondait également à une unité politique. En effet, chaque cho était considéré comme une unité autonome, indépendante les unes des autres. Lorsque des différends existaient parmi ses membres, c'était le comité d'aînés du cho concerné qui prenait la charge de les régler, sans intervention des autres cho. Et quand il surgissait des différends entre les membres de différents cho, les aînés des cho concernés se réunissaient et discutaient ensemble pour résoudre les problèmes.

Nous avons dit que chaque cho était considéré comme une unité autonome, mais cela doit être nuancé, puisqu'il existait une autre organisation hiérarchiquement supérieure qui couvrait plusieurs cho; c'était le nagare, littéralement «le courant», au sens figuré, «le

La fête de gionà Hakata durant l'époque d'Edo

Sur ce tableau du xvil siècle, on peut noter que le char était porté par une centaine d'hommes ; aujourd'hui, du fait de sa plus petite taille, il est porté par une trentaine seulement. boulevard ». L'appelation «boulevard » vient du fait que l'armature géographique et économique de Hakata se composait de sept boulevards, chacun comprenant une dizaine de cho. C'est en s'unissant tous ensemble qu'ils pouvaient préparer la fête onéreuse et exigeante en nombre de participants.

Un nagare ${ }^{5}$ se composait généralement d'une dizaine de cho, donc d'environ deux mille cinq cent personnes. Chaque nagare avait un représentant appelé tsukigyoji ; c'est lui qui dirigeait la réunion des aînés qui, chacun, représentaient leur cho. 
Tous les problèmes importants dépassant le niveau du cho étaient discutés lors de cette réunion. Le bon déroulement des relations entre les cho dépendait donc de cette réunion, et plus particulièrement du tsuki-gyoji. Mais celui-ci était incapable d'imposer son avis aux autres, puisque tous les aînés étaient égaux en qualité et qu'ils occupaient cette place à tour de rôle. En effet, l'appelation tsuki-gyoji signifie littéralement « l'arbitre pour un mois », puisque chacun n'assurait ce poste plus d'un mois.

Pendant l'époque d'Edo, Hakata faisait partie du Kuroda-Han (le territoire du seigneur Kuroda). Celui qui gouvernait Hakata était un samurai dont le poste était appelé machi-bugyo ( l'administrateur de la cité »). Au-dessous de celui-ci, tous les postes étaient assumés par les citadins eux-mêmes. Ils avaient deux chefs, appelés nen-gyoji, « l'arbitre pour une année ». Chaque nen-gyoji assumait ce poste pendant six mois avant dêtre remplacé par un autre. Et l'année suivante, les nouveaux nen-gyoji étaient élus parmi les aînés représentants les nagare. Malgré l'organisation pyramidale officielle dont le but était d'assurer la bonne marche de la vie politique de la cité, l'organisation politique de Hakata reposait, en fait, sur le principe d'égalité et d'autonomie des cho.

\section{La fête en tant quaappareil hégémonique}

Quelle place la fête a-t-elle prise dans la vie des citadins de Hakata ? Cette fête qui exigeait beaucoup de dépenses, a-t-elle empêché le développement économique de la ville? Pourquoi les citadins l'ont-ils exécutée avec tant de zèle et de fidélité ? Voilà les questions auxquelles nous voulons répondre dans cette partie.

Comme nous l'avons remarqué plus haut, la signification religieuse de la fête de gion résidait dans la consécration des chars dans le temple shintoïste, et leur tournée dans la ville pour la débarrasser de tous les mauvais sorts. Le moment crucial avait lieu le 15 juin du calendrier lunaire. De bon matin, avant le lever du soleil, les six nagare ${ }^{6}$ amenaient leur char devant le temple de Kushida. Au signal, à cinq minutes d'intervalle, chacun des six chars entrait dans la cour de Kushida pour y être consacré, avant de faire le tour de la ville. On considérait comme une humuliation qu'un char soit rattrapé par le suivant, c'est ainsi que les participants portaient et poussaient le char de toutes leur forces.

Chaque char devait parcourir cinq kilomètres, porté sur l'épaule par une trentaine de personnes et poussé par une vingtaine d'autres pour le faire avancer le plus vite possible. Il était d'usage, pour chaque nagare, de demander de l'aide aux paysans vigoureux des villages voisins de Hakata.

Ce jour là était certainement le moment de la fête où se manifestait le plus d'enthousiasme; mais ce n'était ni le premier ni le seul jour où les chars circulaient. À partir du premier juin, les habitants de chaque nagare portaient le char pour le faire tourner sur leur territoire, à savoir le boulevard et les rues qui leur appartenaient. Ceux qui avaient des postes à responsabilité devaient assister à la réunion presque chaque jour à partir du premier janvier, afin de préparer et de veiller au bon déroulement des festivités.

Autrefois, les chars étaient immenses : ils faisaient environ quinze mètres de haut et deux mètreset demi de large ${ }^{7}$. Étant donné que les maisons n'étaient pas très hautes, les chars pouvaient être vus, dit-on, des villages alentours d'où les paysans partaient à Hakata pour venir les regarder. 


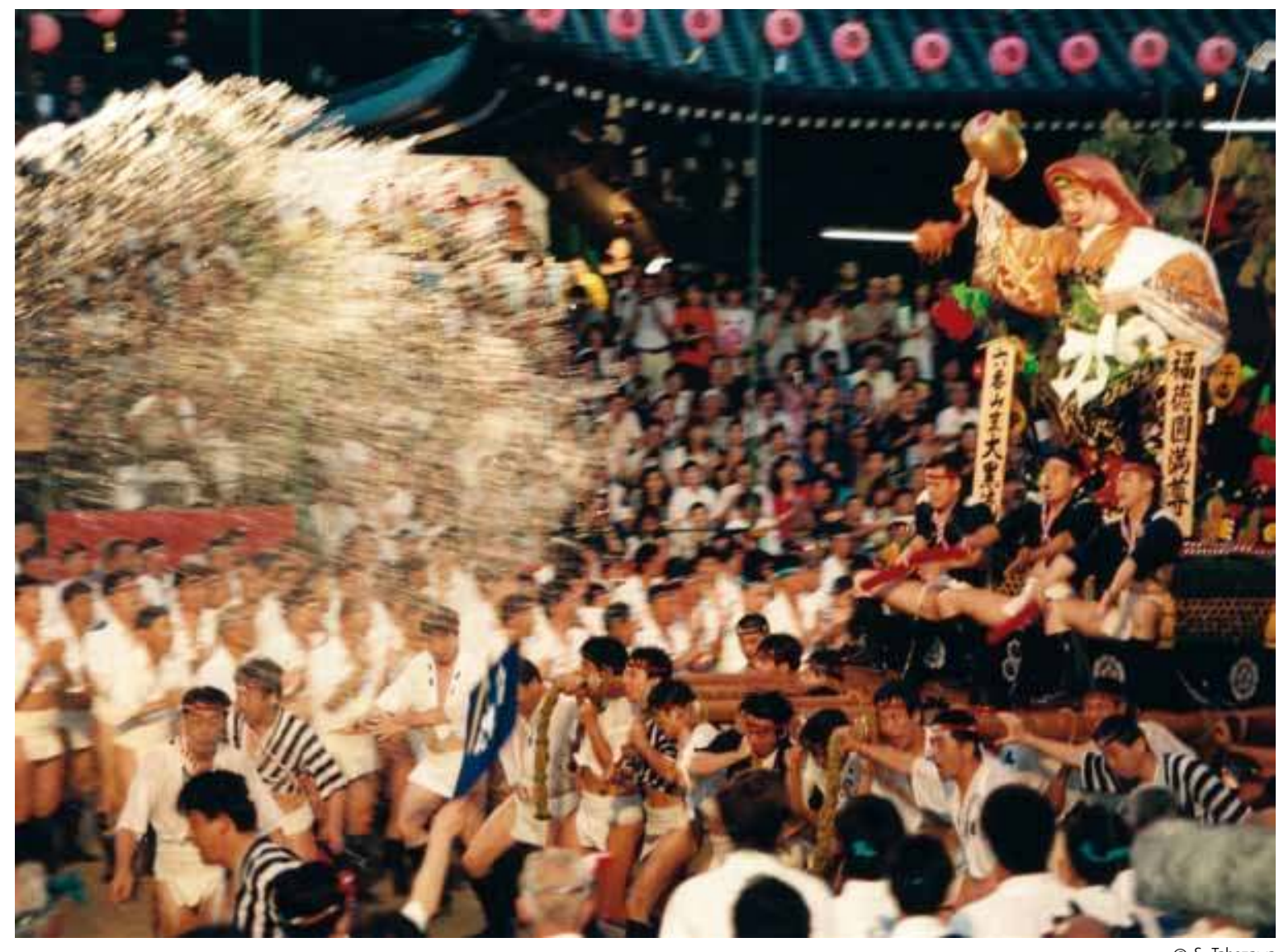

(c) S. Takezawa

Le moment crucial de la fête de gion: l'entrée du char dans le temple shinto de Kushida

Pour encourager ceux qui le portent et ceux qui le poussent, ainsi que pour refroidir leur corps, on projette énergiquement de l'eau sur les participants.
La caractéristique des chars ne résidait pas seulement dans leur taille, mais bien aussi dans leur ornementation ; ils étaient décorés d'une belle manière et de façon dramatique. Un char était considéré comme une sorte de scène ; les poupées ornées de dorures et de soieries étaient arrangées avec des accessoires, de sorte que chaque char représentait une scène du théâtre de kabuki ou un événement historique populaire. Ceux qui réalisaient la décoration du char fabriquaient, pendant leur temps libre, des poupées de Hakata en céramique, très appréciées partout au Japon.

Si la fête était ainsi l'occasion d'une initiation esthétique et physique des citadins, elle était également l'occasion de leur apprentissage social. Quand un enfant naissait dans un des cho de Hakata, il participait à la fête dès son plus jeune âge, porté par son père. Quand il grandissait, il y participait en tant que membre de la classe des enfants : il courrait devant le char avec ses camarades, en portant une planche où figurait le nom du nagare auquel il appartenait. À quinze ans, c'est-à-dire l'âge adulte, il s'engageait en tant que membre à part entière : il aidait à la construction du char, le portait et le poussait lors du défilé ; il servait et desservait le banquet après chaque parcours. Ce rôle revenait aux hommes, car les femmes n'étaient pas autorisées à assister au déroulement de la fête ${ }^{8}$. À vingt-cinq ans, et s'il était considéré comme un personnage sérieux, il occupait le poste dit d'aka-tenogoi. Aka-tenogoi signifie littéralement « la serviette rouge », puisqu'il portait 
toujours la serviette rouge qui le distinguait des autres. Il y avait généralement trois akatenogoi pour chaque cho. C'est eux qui avaient la charge du bon déroulement de la fête. Ils dirigeaient la construction du char, et, au moment du défilé, ils commandaient les autres participants afin que le char circule rapidement et sans accident. Même dans la vie quotidienne, ils donnaient des conseils aux autres membres de leur cho.

Un aka-tenogoi restait à ce poste pendant une dizaine d'années environ. Quand il se mariait et avait des enfants, il perdait ce rang pour entrer dans la classe des aînés. Ces derniers prenaient en charge la bonne gestion du cho, en dressant le budget de la fête. Ils fixaient et collectaient la cotisation de chaque famille, dont le montant dépendait de leur richesse $^{9}$, et ils intervenaient pour résoudre des différends entre cho si nécessaire.

Lorsqu'une personne qui avait fait l'office d'aka-tenogoi mourrait, le char s'arrêtait lors de la fête devant la porte de la maison du défunt pour l'honorer. Ce n'était pas seulement les membres de son cho, mais bien tous les membres du nagare qui chantaient la chanson de la fête, pour rappeler son mérite sa vie durant. C'est ainsi qu'un homme naissait dans le cho, y grandissait et y mourrait. Même aujourd'hui, quand on demande aux gens la raison de leur participation, la plupart d'entre eux répondent que leur vie entière se trouve dans la fête, voire que la fête est leur vie. La fête donnait ainsi un exemple idéal de vie et de mort à tous ses participants. C'était sans aucun doute la principale raison pour laquelle ceux-ci s'y consacraient entièrement.

Nous avons jusqu'ici analysé les divers aspects de la fête de Hakata au temps prémoderne. Nous avons vu que les rôles que cette fête remplissait pour le développement de la vie sociale, physique et esthétique des citadins étaient conséquents tout comme les dimensions économiques.

Si nous étudions les chiffres d'affaires de la ville de Hakata au temps pré-moderne, nous pouvons énumérer les principales mar-

chandises par ordre d'importance : les bougies, les ustensiles en métal, les vêtements, le saké, le shôyu (la sauce japonaise), et l'huile pour la cuisine. La somme de ces six marchandises représentait 81,4\% du montant total des marchandises exportées de Hakata ${ }^{10}$.

La bougie était la spécialité du Kuroda-han qui encourageait tous ses paysans à planter les arbres qui la produisent. Les autres marchandises étaient celles qui étaient consommées dans la vie quotidienne, autant par les citadins que par les paysans.

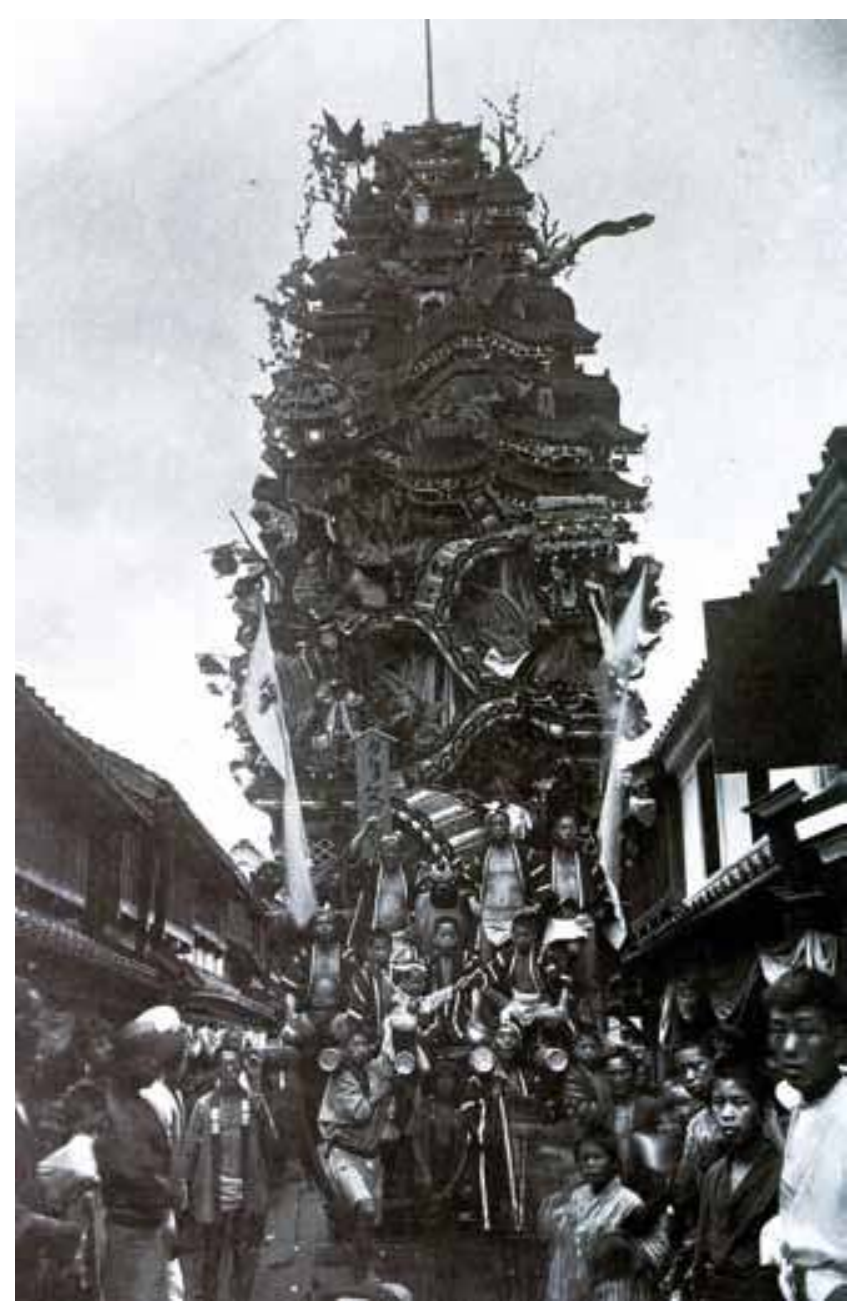

Le char de la fête de gion de Hakata fin xixe siècle

Le char était alors nettement plus élevé que les maisons, et son trajet était alors visible des villages voisins. Ainsi, dès que le char commençait à circuler, les habitants pouvaient se rendre à Hakata pour assister à la fête. 


\section{La poupée de faïence, appelée « Hakata-ningyo», poupée de Hakata \\ Ce type de poupée, fabriquée par ceux qui s'engagent dans \\ la décoration du char est une des poupées les plus appréciées au Japon.}

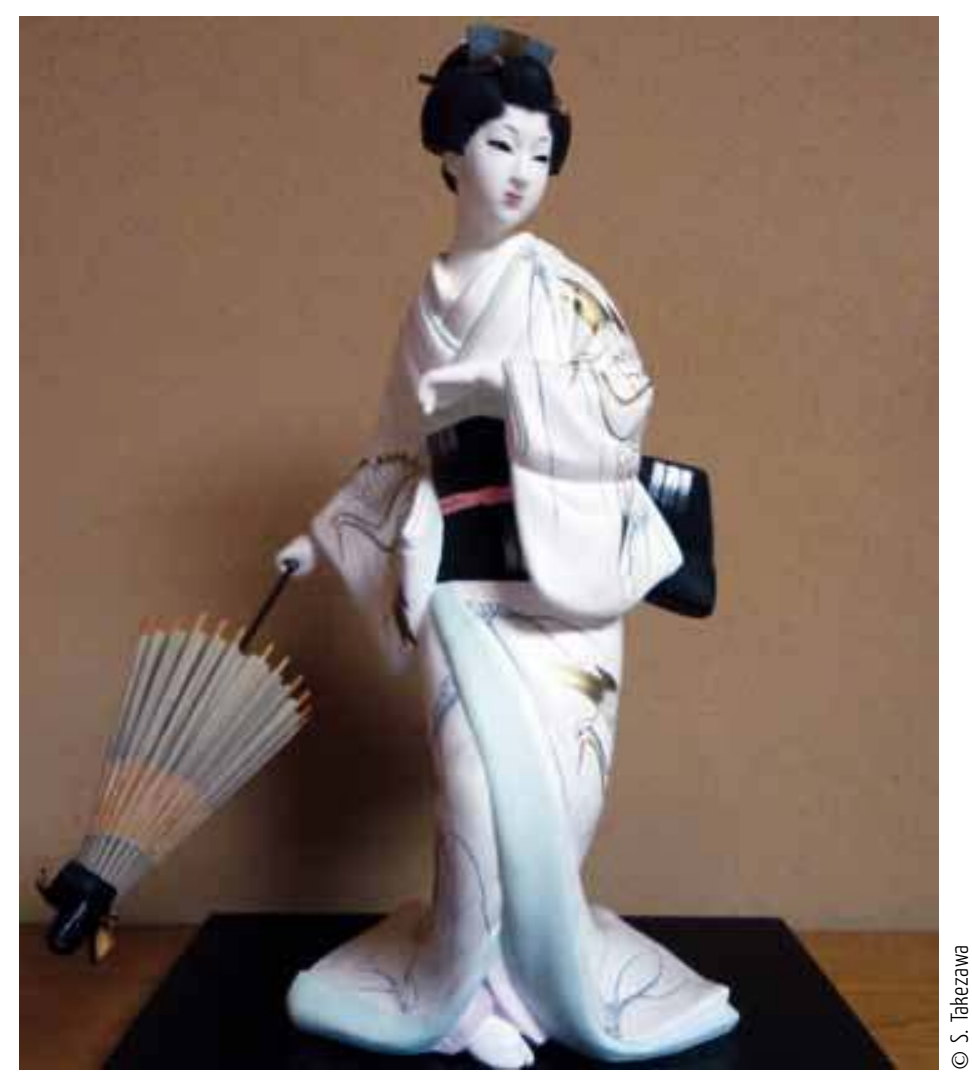

Par ailleurs, ces marchandises étaient utilisées dans la fête. En effet, lors de la fête, les participants portaient des vêtements raffinés en coton dont le motif était différent d'un cho à l'autre. Le char était orné de poupées richement décorées, de feuilles d'or et de soie; à ses quatre pieds était mis un fer pour les stabiliser ; et les citadins, comme les paysans, qui participaient, mangeaient en grande quantité et buvaient beaucoup de saké. On pourrait dire que la fête constituait une sorte de foire, au cours de laquelle on pouvait présenter les spécialités de Hakata et les vendre aux spectateurs.

Si la fête de gion attire aujourd'hui plus d'un million de spectateurs, la fête d'autrefois pouvait, elle aussi, attirer beaucoup de spectateurs, comme l'a écrit un grand savant de l'époque d'Edo, Kaibara Ekken :

La fête de gion se déroule le 15 juin. Ce jour-là, il y a également une représentation du nô. Les citadins fabriquent de grands chars pour les faire circuler dans la ville. [...] Pendant le déroulement de la fête il n'y a pas que les populations alentours, mais bien aussi les hommes et femmes des pays voisins, qui viennent y assister. Ils y descendent pour passer quelques nuits. [...] Une fête comme celle-ci qui attire de si nombreux spectateurs est très rare, même dans les autres pays (Kaibara 1988).

Ce charme, cette force attractive de la fête, étaient appréciés des citadins. Ils la considéraient comme indispensable au développement de leurs activités commerciales. Cependant, à l'époque d'Edo, la classe régnante était celle des samurai, dont le revenu provenait principalement des produits agricoles dont la quantité exploitable avait été fixée à l'avance. L'épargne était la règle absolue du règne des samurai et la classe dominante du Kuroda-han essaya plusieurs fois d'interdire l'exécution de la fête, ou au moins, de diminuer la dépense nécessaire à son exécution. À chaque fois qu'un règlement obligeant à faire des économies était publié, les citadins envoyaient une lettre dans laquelle ils déclaraient que la prospérité de leur cité s'éteindrait si la fête n'avait pas lieu (Hakata Yamakasa Kiroku 1975 : 36, 45).

L'époque d'Edo fut une époque austère. Pendant plus de deux siècles, la classe régnante interdit le libre-échange avec les pays extérieurs. Hakata qui avait été prospère grâce au libre échange avec la Chine et la Corée devint une cité fermée dont les revenus consistaient dès lors dans l'extraction de ressources matérielles des villages et des petites villes alentours. Dans une telle situation, plus la fête devenait somptueuse 
et spectaculaire, plus il y avait de spectateurs et donc de consommateurs, qui venaient y participer. La fête était dans ce sens un appareil hégémonique que la cité marchande de Hakata élaborait pour imposer sa domination culturelle et économique sur les populations voisines ${ }^{11}$.

Durant l'époque d'Edo, la fête citadine japonaise se développa dans la plupart des cités. Il s'agissait sans aucun doute d'une stratégie que les cités choisirent de mettre en place dans le but de maintenir une certaine

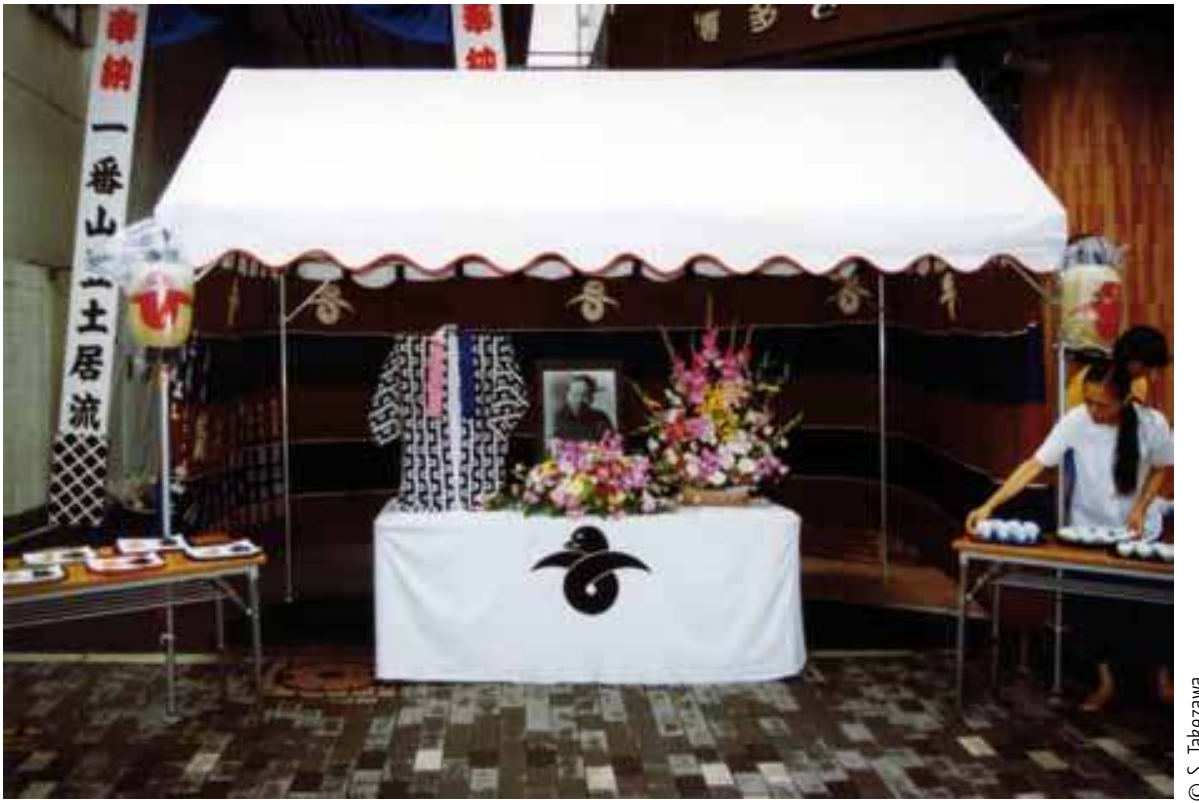
prospérité économique et pour lutter contre le

La fête de gion est également l'occasion de rendre hommage à un défunt mort depuis la dernière fête. Le char s'arrête devant la maison du defunt et tous les participants chantent une chanson de la fête en son honneur.

\section{Les conflits d'interprétation sur la fête à l'époque de Meiji}

En 1868, le shogounat s'effondra et fut remplacé par le gouvernement de Meiji. Ce nouveau gouvernement tenta de centraliser tous les pouvoirs jusqu'alors partagés par les différentes entités sociales : le pouvoir politique était aux mains des samurais, tandis que le pouvoir économique était tenu par les commerçants. Ainsi, au niveau social, le nouveau gouvernement abolit la distinction des classes héréditaires pour recruter les administrateurs en fonction des résultats d'un examen normalisé par l'État. Au niveau économique, le gouvernement créa la banque nationale qui unifia la monnaie et put dès lors surveiller les activités des banques secondaires. Au niveau de l'éducation, il promulgua une loi-cadre en vertu de laquelle toutes les écoles devaient assurer la formation des enfants. Enfin, au niveau religieux, il établit par décret le shintoïsme étatique, auquel tous les Japonais devaient adhérer, dans le but d'instaurer une conscience nationale et d'empêcher la pénétration des religions étrangères, tel que le christianisme. Tous les temples furent organisés de manière hiérarchique les uns par rapport aux autres, et non considérés comme égaux et équivalents, ce qui était le cas auparavant. 


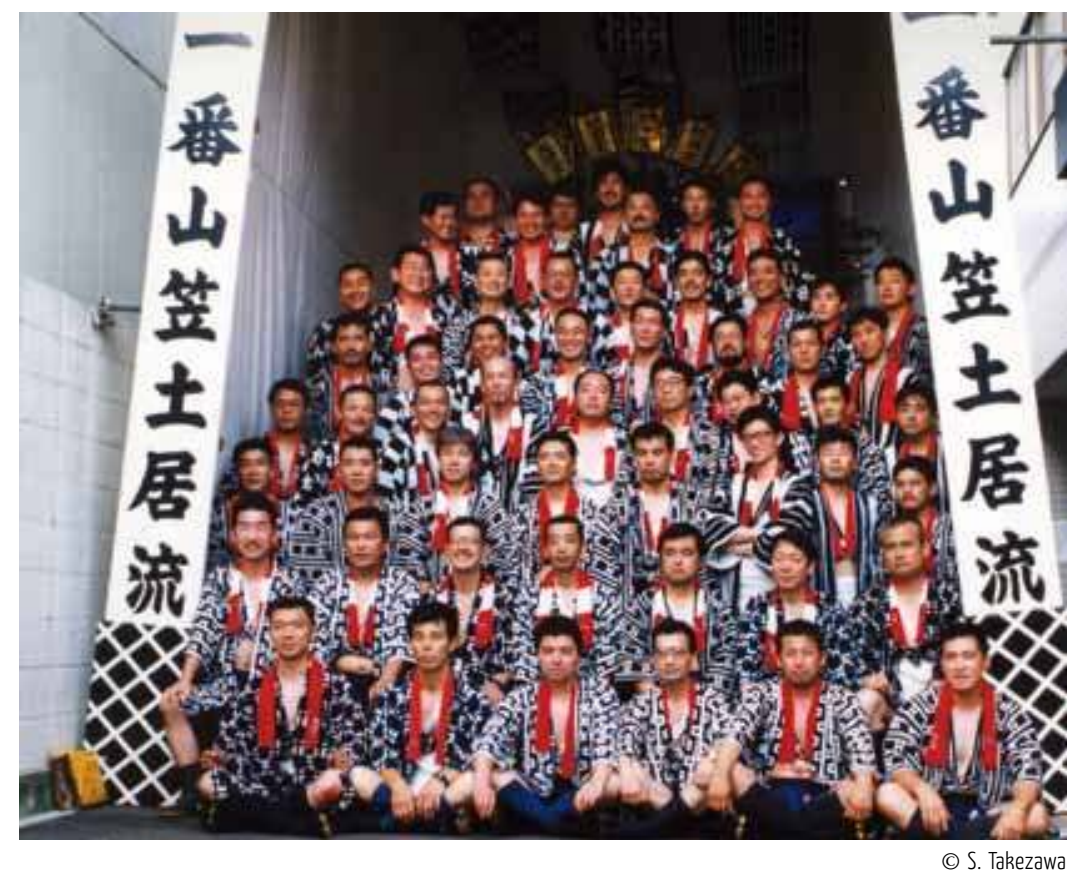

L'assemblée des commissaires de la fête de gion

Tous les aka-tenogoiet tous les aînés du nagare concerné présents devant le char.
Il uniformisa les rituels effectués dans tous les temples shintoïstes et les prêtres des temples shintoïstes devinrent des fonctionnaires rétribués par l'État (Murakami $1970: 118$ sq.).

Au cours de ce processus de centralisation, la fête de gion fut interdite trois fois. Néanmoins, chaque fois qu'elle fut interdite, les citadins de Hakata protestèrent vivement, de sorte que la fête reprenait peu après. Nous pouvons remarquer une grande différence autant entre les arguments soutenant l'interdiction de la part du gouvernement central et/ou local et ceux soutenant la contestation de la part de la société locale qu'entre ceux de la première interdiction et ceux de la deuxième ou de la troisième interdiction. La confrontation de ces arguments nous sera utile pour faire la lumière sur la nature et l'évolution de la relation entre ces deux entités sociales. Tel est ici l'objectif de notre analyse.

En 1872, quatre ans après la restauration de Meiji, la fête de Hakata fut la cible de la première interdiction par le gouverneur nouvellement arrivé. Avant la restauration de Meiji, c'était le chef des samurai qui gouvernait le han (la préfecture); ce poste était transmis de manière héréditaire. Comme il était proche de la population de Hakata, il continuait d'autoriser l'exécution de la fête quoiqu'il essaya à plusieurs reprises de lui faire faire des économies. En revanche, le nouveau gouverneur, envoyé par le gouvernement central, était étranger à la société locale, si bien qu'il pensa mener sa politique uniquement d'après les directives du gouvernement central. Ainsi, sous prétexte que la fête était une dépense inutile et qu'elle était une coutume sauvage qui ne s'accordait plus à la nouvelle ère que le gouvernement de Meiji souhaitait, il annonça officiellement son interdiction. Cette interdiction refléta, en réalité, l'intention du gouvernement de contrôler toutes les activités religieuses pour mieux instituer le shintoïsme étatique.

À l'encontre de cet avis officiel, la population locale envoyait chaque année une lettre de pétition pour obtenir l'autorisation de l'exécution de la fête. Ces lettres expliquaient que la fête était « destinée à obtenir la grâce divine et à expulser de la cité tous les mauvais sorts $»^{12}$; elles affirmaient que la fête locale s'accordait parfaitement avec le principe du shintoïsme étatique. Néanmoins, elles restèrent vaines jusqu'en 1883, période où nombre de Japonais souffrirent de l'augmentation des impôts et de l'abaissement du niveau de vie, conséquence de la politique déflationniste du gouvernement. À la suite de manifestations et de grèves partout dans le pays, le gouvernement décida de modifier sa politique stricte en matière de religion, estimant que la fête locale servirait de soupape aux mécontentements de la population. 
La fête de gion reprit donc en 1883, mais elle fut de nouveau l'objet d'une interdiction en 1897. Cette fois-ci, pourtant, l'interdiction ne vint pas de l'extérieur, mais de l'intérieur de la cité, de la part des grands entrepreneurs, tel que les banquiers et les patrons des maisons du commerce extérieur. Ceux-ci prétendirent que la fête de gion risquait de couper la ligne électrique qui venait d'être installée dans la ville cette année-là. Ils prétendirent également que la fête, réalisée par des hommes presque nus, n'était que la manifestation d'une barbarie qui entachait la réputation de la cité. Ils exercèrent une pression telle sur les conseillers municipaux, que ceux-ci votèrent l'interdiction de la fête juste avant de son déroulement (Hakata Yamakasa Kiroku 1975 : 98).

Pour comprendre l'arrière-plan de cette mauvaise intention de la part des grands entrepreneurs, il faut jeter un œil à l'évolution économique japonaise de l'époque. À la suite de la guerre contre la Chine en 1894-95, le Japon reçut une indemnité considérable. Le gouvernement japonais choisit d'investir cette somme dans un secteur productif, l'industrie, en vue d'opérer sa révolution industrielle. Le résultat fut favorable. Entre 1895 et 1904, le nombre des entreprises tripla, le montant total des capitaux de toutes les entreprises japonaises quadrupla, et la production du fil de coton fut multiplié par trois (Inoue 1968 : 96). Ainsi fut franchie la première étape de la révolution industrielle. Les entrepreneurs de la cité de Fukuoka ne voulurent pas regarder avec indifférence cette vague favorable au développement économique. Ils s'engagèrent alors dans la modernisation du secteur économique : en organisant la première grande foire qui couvrit toutes les îles de Kyushu en 1887, et en construisant un chemin de fer entre Fukuoka et Tsikugogawa, en 1889.

La différence entre la première et la seconde interdiction apparaît nettement. La première vient de l'extérieur, au nom d'un gouverneur envoyé par le gouvernement central. En raison du shintoïsme étatique que le gouvernement tentait de constituer, ce gouverneur critiqua alors les pratiques locales qui dépensaient, d'après lui, trop de richesses.

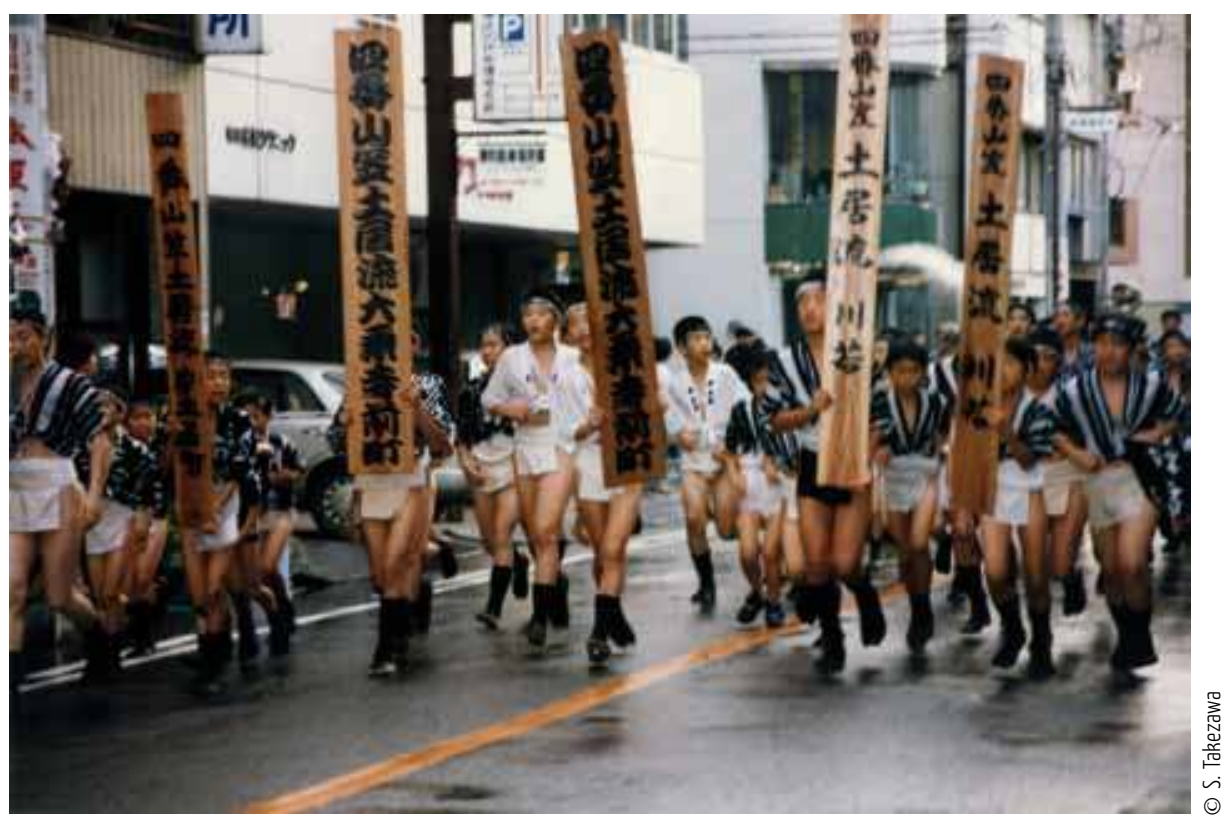

\section{Les enfants participent eux-aussi à la fête \\ Jusqu'à leur 18 ans, les enfants courent devant le char, en portant des plaques représentant le cho auquel ils appartiennent.}


Chaque cho a une lanterne spécifique. De nos jours, la lanterne a été remplacée par une lampe électrique, qui conserve toutefois le signe distinctif propre à chaque cho.

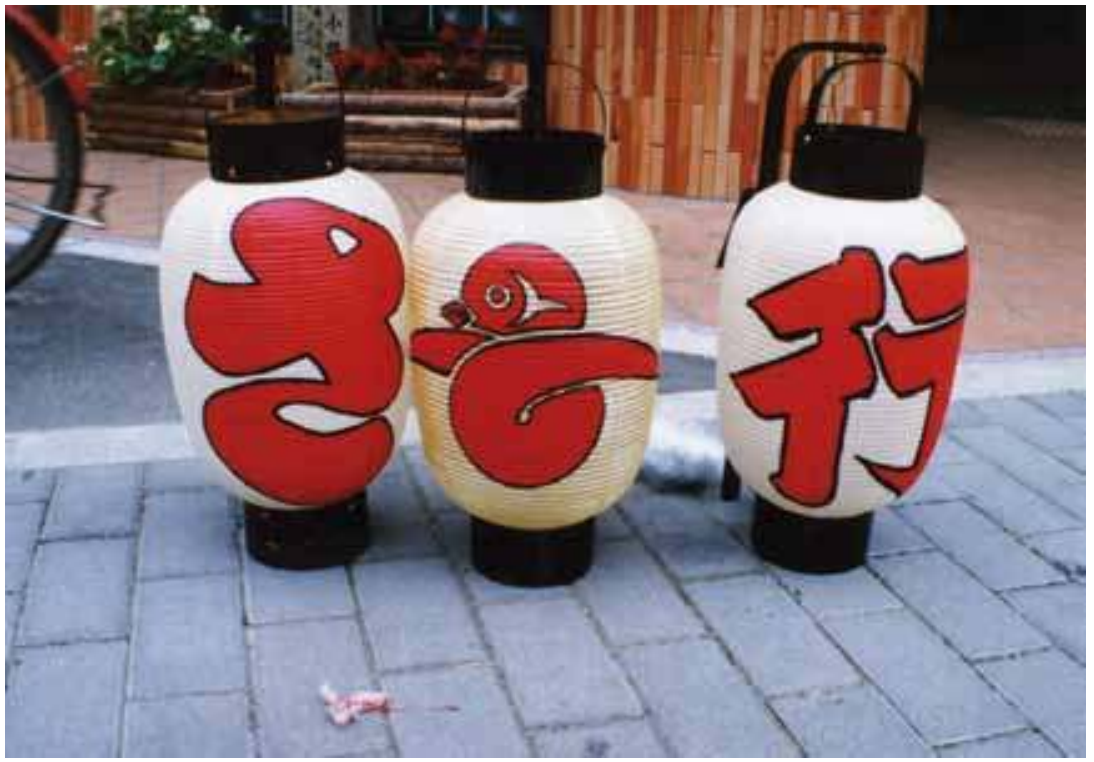

(c) S. Takezawa tramway (Quotidien de Fukuoka, 10/6/1910).
Face à cette critique, les citadins insistèrent sur l'objectif de la fête, à savoir de respecter le dieu ; ils prétendirent ainsi que leurs pratiques étaient adéquates, au moins admissibles, puisqu'elles n'étaient pas en contradiction avec le principe du shintoïsme étatique.

Mais ce ne fut pas le cas pour la deuxième interdiction. Cette fois-ci, c'était la logique de la modernisation qui avait poussé les grands entrepreneurs et les conseillers municipaux à agir contre la fête traditionnelle. Face à cette logique, l'argument traditionaliste qui avait été avancé contre la première était, bien entendu, inefficace. Embarrassés par cette impuissance, les citadins de Hakata décidèrent de recourir à un journaliste (Furushima Kazuo) nouvellement arrivé de Tokyo pour les conseiller. En observant de près la réalité de la fête, celui-ci énuméra ses mérites. Il remarqua que le cho, en tant qu'unité de base de la fête, servait également de fondement à l'administration locale, puisqu'il collectait les impôts. Il donna ensuite comme conseil aux participants de porter des vêtements courts plutôt que d'être à demi-nu, afin d'éviter la critique de la barbarie de la fête. Cet argument fut un succès. Il convainquit les conseillers municipaux comme la population locale. L'interdiction votée au conseil municipal fut annulée et la fête put continuer, à condition d'opérer quelques modifications.

Pourtant, l'hostilité envers la fête de gion surgit de nouveau en 1910. Cette fois, l'interdiction, ou plus exactement la proposition d'un changement total des modalités du déroulement de la fête, fut de nouveau avancée de l'intérieur. Le maire de la cité de Fukuoka ${ }^{13}$ exprima officiellement le peu d'estime qu'il avait pour la fête :

La fête de gion doit-elle continuer encore ? Même s'il est vrai qu'elle contribue à attirer les spectateurs extérieurs, est-il possible de faire face à son énorme dépense? Aujourd'hui, le tramway qui peut transporter les étrangers s'est installé. Je me permets donc de proposer l'abolition de la fête, ou le cas échéant, d'interdire la tournée des chars dans la ville, afin d'éviter la coupure de la ligne électrique du 


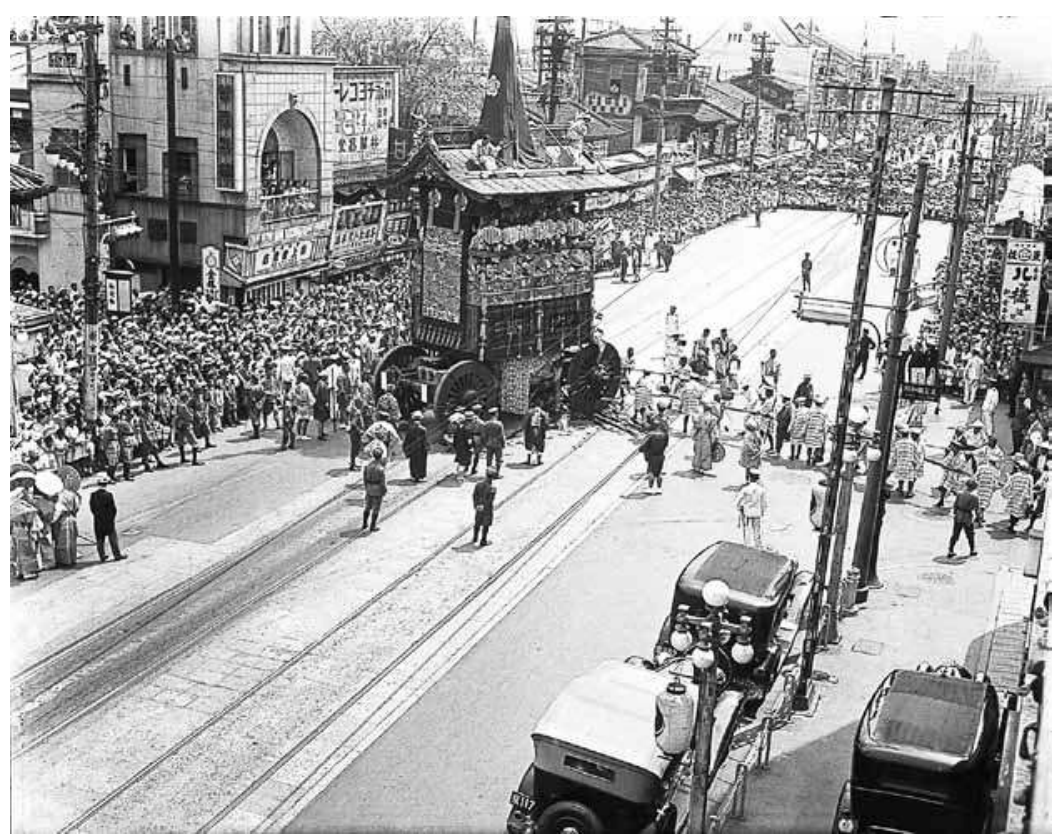

(C) archives CNRS-MAE/fonds Leroi-Gourhan
Fête de gion de Kyoto prise par Leroi-Gourhan dans les années 1930

La fête de gion de Hakata a commencé sous l'influence de celle de Kyoto. On peut aujourd'hui voir une différence considérable entre ces deux fêtes, tant au niveau des modalités de l'exécution qu'au niveau de l'organisation.

fête et d'inventer de nouvelles modalités susceptibles d'attirer plus de consommateurs. Mais ces propositions eurent un effet autre et plus profond, qui touchait directement au principe même de la société moderne. Au fond, ce qu'elles remettaient en question n'était pas la logique économique, mais la logique d'administration de la cité, autrement dit, la logique touchant à l'organisation publique.

Comme nous l'avons vu plus haut, c'était le cho et le nagare, en tant qu'unités de base de la fête, qui maintenaient, au temps pré-moderne, l'infrastructure de la cité, à savoir les rues, les boulevards et les ponts. C'est ainsi que les citadins groupés dans le cho pensaient garder le droit usufructuaire du boulevard et des rues qui faisaient partie de leur territoire. Et pourtant, depuis que la municipalité avait investi une somme importante dans l'installation du tramway le long des boulevards, elle considéra qu'ils appartenaient au bien public et que le droit usufructuaire des territoires n'était plus dans les mains de la société locale, mais bien dans celle de la municipalité. C'est ainsi que la municipalité et la police intervinrent dans l'exécution de la fête pour faire en sorte que la procession des chars ait lieu dans un parc.

Malgré ces modifications, la fête de gion put continuer de se réaliser. Mais cela s'accompagna de la diminution de la hauteur des chars qui était désormais fixée à deux mètres, afin d'éviter la coupure de la ligne électrique du tramway. Dorénavant, la fête ne devait plus être organisée de façon autonome mais conformément aux directives de la politique gouvernementale militarisée de l'époque. De fait, en 1904, pendant la guerre contre la Russie, les citadins de Hakata décidèrent de réduire le nombre des chars à un seul, dans le but d'apporter une contribution pour aider au financement de la guerre. En 1938, quand la délégation italienne visita Hakata pour commémorer le pacte tripartite, ils réalisèrent, en signe de bon accueil, la tournée des chars en mars, pour la première fois dans la longue histoire de la fête de gion (Hakata Yamakasa Kiroku 1975 : 99). 


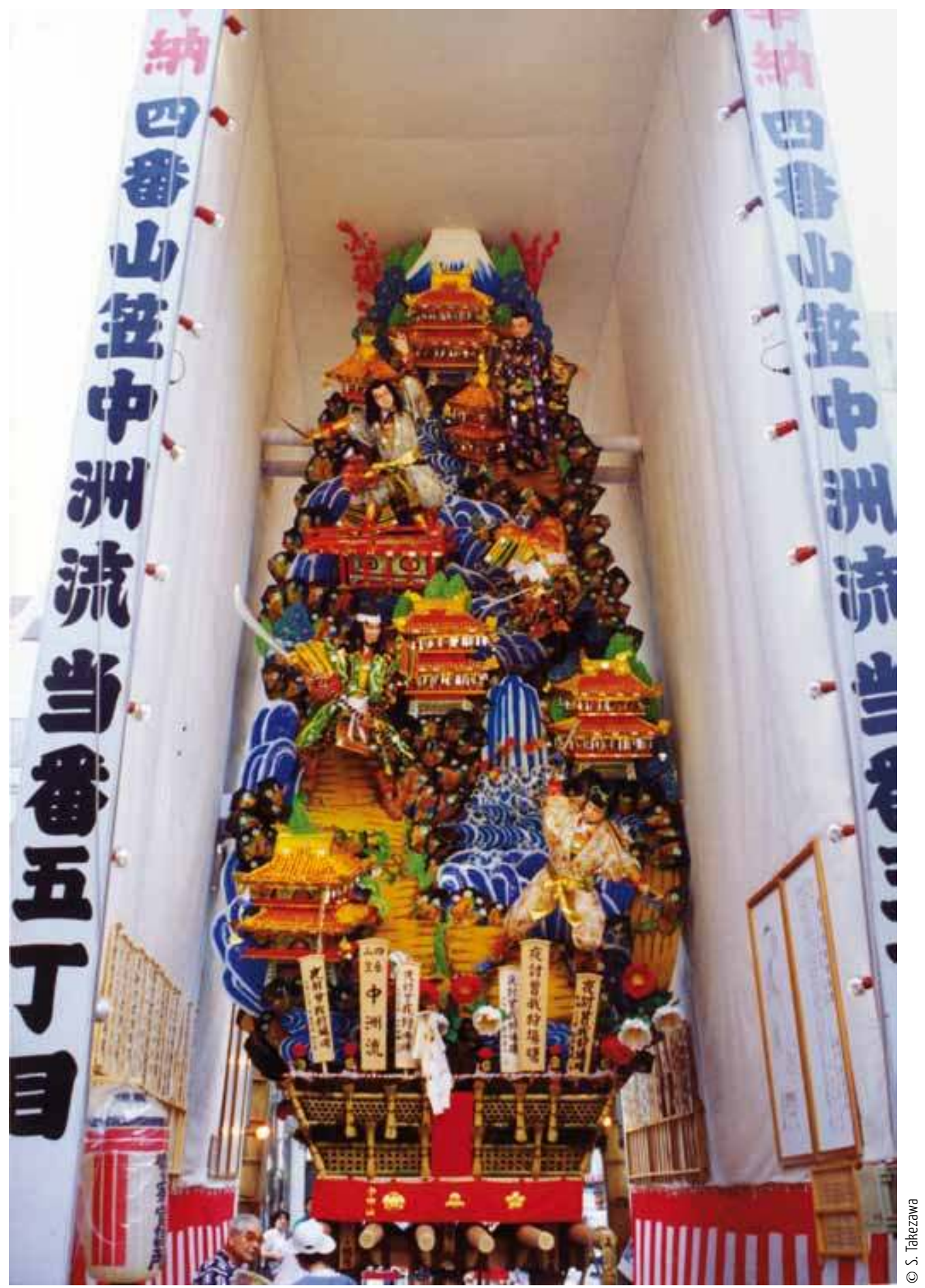

\section{Ce type du char est appelé kazari-yama}

II s'agit d'un type traditionnel de char dont la hauteur est de quinze mètres. Depuis que la ligne de tramway a entouré la ville, ce char ne circule plus. Aujourd'hui, néanmoins, une dizaine de chars de ce type sont construits et jouent encore un rôle lors de festivités. 
Si l'on étudie de près l'évolution de la fête de Hakata, il est possible d'y reconnaître les traits caractéristiques de la profonde transformation du système social appelée « modernisation» qui frappa toute la société japonaise. Au cours de ce processus, le gouvernement s'empara de toutes les fonctions que la société locale avait gardées au temps pré-moderne. Avant la mise en route de ce processus de modernisation, c'était par les cho concernés que l'infrastructure de la cité pouvait se maintenir ; désormais, c'était à la municipalité que revenait la charge d'assumer le maintien et la réorganisation de ces infrastructures. Auparavant, les enfants et les jeunes de la cité recevaient leur formation esthétique, physique et sociale au cours de leur participation à la fête ; désormais, cette fonction revenait aux écoles dites publiques. Avant, la prospérité de la cité était réalisée par la fête, en tant qu'appareil hégémonique imposant sa domination économique sur les villages et les villes alentours; dès lors, la prospérité de la cité ne pouvait être réalisée que par un énorme investissement de la part du gouvernement qui installa le tramway et construisit des usines d'État.

Ainsi, la société locale, qui avait gardé une sorte d'autonomie, perdit toute son indépendance envers le gouvernement central. Elle ne subsistait qu'en tant que province de l'État tout puissant. Et la population locale qui avait alimenté le sens fort de la collectivité en réalisant la fête transférait cette signification à l'État.

\section{$\&$}

En 1941, à la veille de la participation japonaise à la seconde guerre mondiale, un fameux folkloriste japonais, Yanagita Kunio, donna une série de conférences à l'université de Tokyo sous le titre «La fête japonaise ». À la fin de ses conférences, il mis l'accent sur la notion de « collectivité » vécue de façon très forte par les Japonais, qui était alimentée, d'après lui, par la réalisation de la fête :

Je pense que la « reconnaissance » était le principe le plus important de la vie religieuse au Japon, pays divin et incomparable dans le monde. Il va sans dire que toute la population japonaise doit témoigner un respect infini envers les grands temples shintoïstes vénérés par la famille impériale. D’un autre côté, celle-ci approuva officiellement, sans exception, tous les temples shintoïstes vénérés par le peuple japonais, envoya un messager et fit consacrer un édit par un de ses administrateurs aux temples les plus importants. [...] C'est par là que l'unification de la culture dite mentale de notre nation s'est réalisée (Yanagita $1962:$ 311-312).

Il n'est pas difficile d'indiquer le contresens intentionnel de Yanagita, puisque nous savons maintenant que l'unification de la culture et de la mentalité chez les Japonais est née de la mise en place du shintoïsme étatique. La conformité de toutes les pratiques religieuses des Japonais qui a rendu l'unification possible n'était pas quelque chose de traditionnel comme Yanagita voulut le démontrer, mais quelque chose imposé, voire inventé par le shintoïsme de l'époque Meiji. 
Aujourd'hui, les fêtes citadines japonaises, y compris la fête de gion de Hakata, sont extrêmement appréciées. Non seulement elles attirent de nombreux spectateurs et participants, mais elles sont aussi considérées comme des facteurs facilitant la vie collective au sein d'une population qui devient plus en plus individualiste. Et pourtant, d'un autre côté, il y a beaucoup de Japonais, qui ressentent de l'aversion pour ce phénomène, avançant que la fête faisait et fait encore partie d'un shintoïsme qui n'a pas encore officiellement regretté son passé discutable de shintoïsme étatique et de coopération à la guerre d'invasion.

Dans le contexte actuel de la mondialisation, l'importance de la société locale est de plus en plus reconnue. La fête japonaise traditionnelle est ainsi réévaluée en tant que facteur essentiel de la notion de collectivité, réclamée par la population locale. Nous admettons ce rôle crucial que la fête peut et pourra remplir dans la vie sociale et religieuse des Japonais. Mais cela est à condition que le shintoïsme soit reconnu comme religion locale, soutenue par la société locale. 


\section{NOTES}

Photo d'ouverture : La fête de gion à Hakata. La fête de gion a commencé à Kyoto en 869. Réalisée par les plébéiens dès le début, puis transmise durant tout le Moyen-Âge, dans beaucoup de cités japonaises où les citadins continuent de la pratiquer de nos jours.

1. À cette époque, les Japonais prononçaient [fa] au lieu de [ha] comme c'est le cas aujourd'hui, de sorte que Frois écrivit « Facata ». Sakai est une autre cité commerçante japonaise qui était réputée pour son autonomie politique et économique.

2. Le cho correspondait au mura dans la campagne japonaise d'autrefois. Le cho et le mura constituaient la base de la vie sociale et matérielle du peuple japonais. Ils pouvaient jouir de plus ou moins d'autonomie alors que la classe dominante se contentait d'en collecter les impôts. Un mura se composait généralement, comme un cho à Hakata, de deux cent cinquante personnes. C'était sans doute un nombre convenable pour que les gens puissent vivre en commun.

3. Il n'y avait - et il n'y a - qu'un temple shintoïste (Kusida-jinja) dans la ville de Hakata. Les citadins construisirent des magasins pour le stockage du riz sur le terrain de ce temple. Ce fait atteste que la vie économique et la vie religieuse des citadins étaient étroitement liées l'une à l'autre et que le temple shintoïste était considéré comme leur bien public.

4. Cette porte marquant la limite de chaque cho fut supprimée en 1741. Depuis cette époque, la porte est maintenue uniquement à l'extérieur de Hakata. Cette suppression avait pour objectif de permettre une libre circulation de nuit, à Hakata. Elle marquait ainsi l'évolution de la conscience des habitants de la ville : avant, ses habitants menaient la vie communale au niveau du cho; dès lors, ils la menaient au niveau de la ville entière.

5. On ne connaît pas l'origine du nagare. Il existe une description en 1568 selon laquelle il y aurait douze chars de la fête de gion (Hakata Yamakasa Kiroku 1975 : 34); il se peut que le nagare, en tant qu'unité religieuse de réalisation de la fête, ait existé cette année-là. Mais c'est Toyotomi Hideyoshi qui l'organisa, ou réorganisa, en faisant construire les sept boulevards à la fin du Xvi ${ }^{\mathrm{e}}$ siècle.

6. Les sept nagara participaient à la fête, mais l'un d'entre eux prit la responsabilité de faire jouer le nô afin de calmer le dieu, qui aurait pu être excité par le défilé exalté des chars, portés par les six autres nagara.

7. Quand les tramways commencèrent à circuler dans la ville du Hakata au début du $x^{e}$ siècle, la hauteur des chars fut fixée à deux mètres pour éviter qu'ils ne coupent les lignes électriques. Depuis ce jour, la hauteur des chars est restée fixe, même jusqu'aujourd'hui où les tramways ne circulent plus.
8. Les femmes avaient (et ont, aujourd'hui encore) l'interdiction d'y assister, considérées comme porteuses d'impureté à cause de leur menstruation. Tout ce qu'elles pouvaient faire était de regarder la tournée des chars. Néanmoins, cette interdiction avait une fonction économique. Pendant que leur mari s'occupait de la fête, c'était les femmes qui tenaient leur magasin de commerce. Ainsi les grands commerçants de Hakata étaient-ils (et sont-ils, encore) souvent des femmes.

9. Ce fait entraîna une sorte de hiérarchie entre les membres du cho, qui étaient en principe égalitaires. En fait, celui qui payait le plus, avait, dit-on, plus de chance de devenir aka-tenogoi ou tuki-gyoji - respectivement responsables du cho et du nagare. Tous les cho étaient en principe égaux, mais ceux qui payaient plus que les autres avaient plus de chance de diriger les autres cho. Leur domination ne concernait pas seulement la vie religieuse, mais bien aussi la vie politique, puisque les aînés d'un cho riche avaient plus de chance de devenir tsuki-gyoji ou nen-gyoji - à savoir de diriger la politique de la cité.

10. C'est le chiffre des premières années de l'époque de Meiji (1874), puisque nous ne pouvions pas, malgré nos efforts, trouver les chiffres exacts pour l'époque d'Edo. Nous ne pensons pas qu'il y ait de grandes différences parmi les principales marchandises exportées de Hakata entre l'époque d'Edo et les premières années de l'époque de Meiji, la révolution industrielle japonaise n'ayant commencé qu'aux dernières années du XIX ${ }^{e}$ siècle.

11. La fête de gion de Hakata exerçait une influence importante sur les villages et petites villes alentours, dont l'aire d'influence décrivait un cercle d'une cinquantaine de kilomètres de diamètre. Ces derniers imitaient parfaitement la fête de gion de Hakata.

12. Hakata Yamakasa Kiroku, 1975 : 85. Nous y comprenons que les gens de Hakata gardaient le même sens religieux qu'à l'époque d'Edo, alors que le gouverneur essayait d'imposer un nouveau message culturel.

13. Durant l'époque d'Edo, il existait deux cités juxtaposées : une était Hakata, la ville des commerçants, et l'autre Fukuoka, ville fondée autour d'un château. En 1889, ces deux cités fusionnèrent pour former une cité dont le nom se fixa sur Fukuoka à la suite du vote des conseillers municipaux. 


\section{REEFERENCES}

Fróis, L. 1976 Hitoria de Japam, 5 volumes, Biblioteca Nacional de Lisboa.

Hakata Yamakasa Kiroku 1975 (Documents sur la fête de gion de Hakata) Hakata Gion Yamakasa Shinkokai. Inoue, M. 1968 Nihon Teikokushugi no Keisei (Formation de l'impérialisme japonais). Iwanami-shoten.

Kawazoe S. (dir.) 1988 Hakata, Chusei no Shogyo toshi (Hakata, une cité marchande au Moyen Âge),

Yomigaeru Chusei, vol. 1 (Le moyen âge renaissant, I). Heibon-sha.

Murakami, J. 1970 Kokka shinto (Shintoïsme étatique). Iwanamishoten.

Yanagita, K. 1962 (1941) Nihon no Masuiri (La Fête japonaise), Oeuvres Complètes, tome 10: 153-314, Tsikuma-shobo.

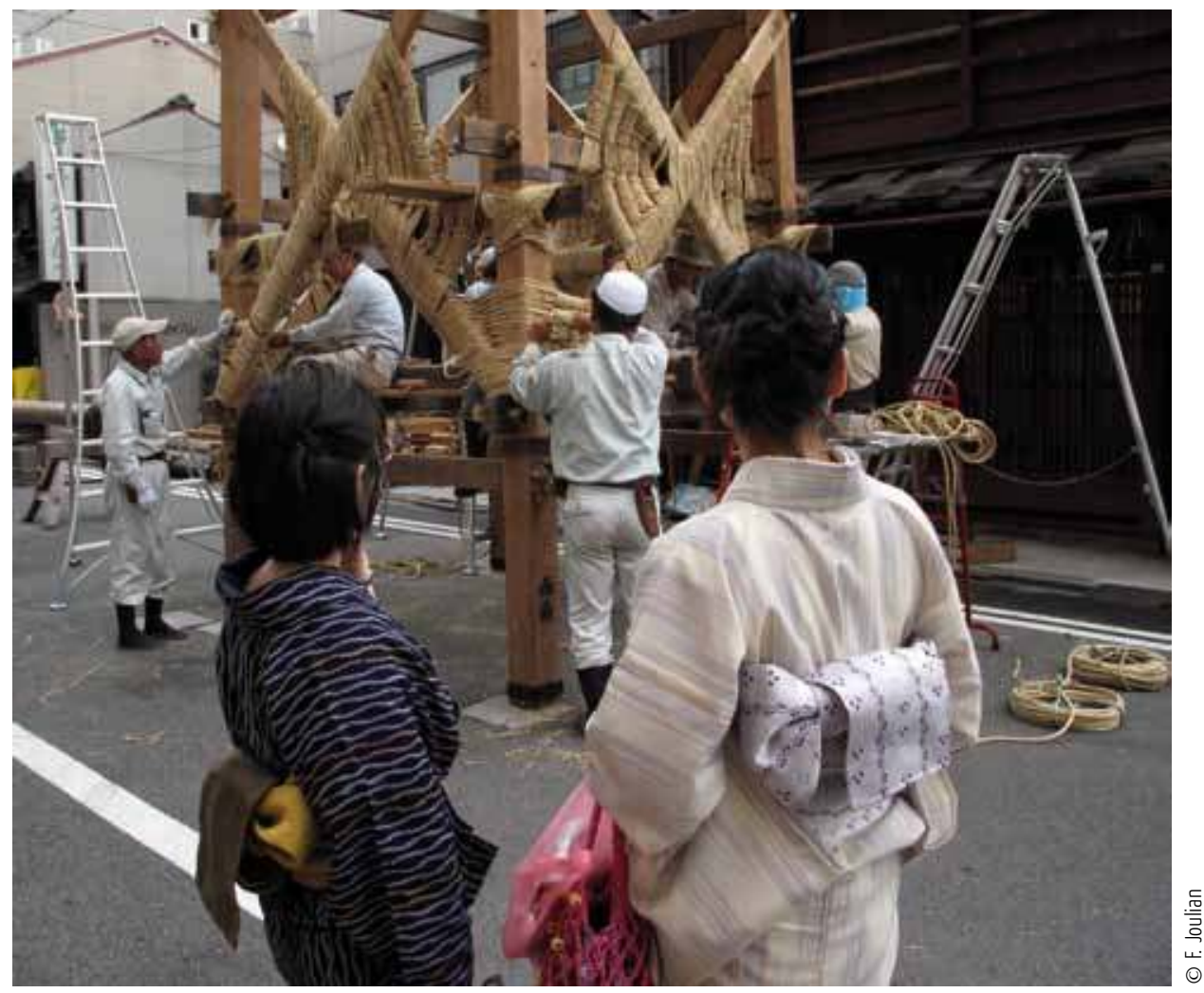

\section{Le montage d'un char de la fête de gion dans les rues de Kyoto, 2009}

Les clous étant proscrits, le montage est fait entièrement avec des cordages. 


\section{RÉSUMÉ}

La fête japonaise: appareil hégémonique de la cité marchande. La vie religieuse de beaucoup de Japonais est axée sur les fêtes shinto. Ces fêtes se multiplient et se diversifient, mais les plus importantes sont sans aucun doute les fêtes citadines d'été, effectuées par des centaines de participants devant plusieurs milliers de spectateurs. Cet article analyse une de ces fêtes, la fête de gion de Hakata ; nous essayons de comprendre comment ses habitants l'ont progressivement développée et rendue plus attractive.

Hakata est une cité marchande depuis environ quinze siècles. Par sa position géographique privilégiée (face à la Péninsule Coreénne), Hakata réalisait sa prospérité économique grâce au commerce extérieur. Mais la politique d'isolement imposée par le shogunat d'Edo l'obligea à inventer une nouvelle mécanique pour manitenir sa prospérité. Elle décida alors d'amplifier sa fête qui devint plus belle et plus spectaculaire, si bien que de plus en plus de spectateurs venaient y assister et y dépensaient beaucoup en achetant divers produits. La fête devint ainsi le moyen par lequel Hakata imposa sa domination économique et culturelle sur les régions environnantes.

Après la restauration de Meiji, le gouvernement entra dans une ère de modernisation économique et culturelle, et intervint plusieurs fois dans l'exécution de la fête. Mais les habitants de Hakata repoussèrent ces assauts pour continuer à réaliser, jusqu'à aujourd'hui, leur fête favorite.

\section{ABSTRACT}

The japanese festival: hegemonic device of the haggle town. The religious lives of many Japanese tend to center around the Shinto festivals. There are many and various kinds of festivals. The most important and most gorgeous are the city festivals that are carried out each year by thousands of citizens before the several hundred thousand spectators. This article analyses one of these festivals, festival of Hakata, to understand how its citizens have developed it to make it more attractive.

In front of the Korean Peninsula, Hakata is one of the oldest cities in Japan. Because of its geographical position, Hakata has been very prosperous through the foreign trade. But the national isolation policy during the Edo period forced her to invent some mechanism to keep its prosperity under such a condition. She decided to glorify its festivals. Really, more the festivals became splendid, more spectators visited her to buy merchandises there. Thus, the festival became the instrument by which the city of Hakata dominated the surrounding towns and villages to obtain the human and material resources.

After the Meiji Restoration, the central and local governments intervened three times to prohibit her festivals. But the citizens of Hakata thrust the prohibitions to continue her festivals up to now.

\section{MOTS CLÉS}

Fête, shintoïsme, cité médiévale, hégémonie, modernisation, politique religieuse

\section{KEYWORDS}

Festival, shintoïsm, commercial city, hegemony, modernization, religious policy 